\title{
Current-voltage characteristics of graphene devices: Interplay between Zener-Klein tunneling and defects
}

\author{
Niels Vandecasteele, ${ }^{1}$ Amelia Barreiro, ${ }^{2, *}$ Michele Lazzeri, ${ }^{1}$ Adrian Bachtold, ${ }^{2}$ and Francesco Mauri ${ }^{1}$ \\ ${ }^{1} I M P M C$, IPGP, Universités Paris 6 et 7-CNRS, 140 rue de Lourmel, 75015 Paris, France \\ ${ }^{2}$ CIN2, CSIC-ICN, Campus UAB, E-08193 Barcelona, Spain
}

(Received 2 October 2009; revised manuscript received 14 May 2010; published 20 July 2010)

\begin{abstract}
We report a theoretical/experimental study of current-voltage characteristics $(I-V)$ of graphene devices near the Dirac point. The $I-V$ can be described by a power law $\left(I \propto V^{\alpha}\right.$ with $\left.1<\alpha \leq 1.5\right)$. The exponent is higher when the mobility is lower. This superlinear $I-V$ is interpreted in terms of the interplay between Zener-Klein transport, that is tunneling between different energy bands, and defect scattering. Surprisingly, the Zener-Klein tunneling is made visible by the presence of defects.
\end{abstract}

DOI: 10.1103/PhysRevB.82.045416

PACS number(s): 72.80.Rj, 72.10.Fk, 73.50.Dn, 73.61.Wp

\section{INTRODUCTION}

Zener tunneling ${ }^{1}$ is a concept known since the 30 s, which, in a solid, refers to the tunneling of carriers from one band to another through the forbidden energy gap (for example, from the conduction to the valence band). This tunnel process is very intriguing in graphene because the energy gap is suppressed to zero and because of the peculiar charge carriers behaving as Dirac fermions., ${ }^{2,3}$ In particular, some of the carriers (those with the velocity parallel to the electric field) experience Zener tunneling without being backscattered, ${ }^{3-6}$ a behavior which is markedly different from the one in conventional semiconductors. The physics is the same as for relativistic electrons tunneling through a barrier, a phenomenon called Klein tunneling ${ }^{7}$ and, for this reason, we will use the term Zener-Klein (ZK) tunneling.

In view of the remarkable properties of $\mathrm{ZK}$ tunneling in graphene, it is understandable that an intensive endeavor was made to challenge it. So far, the effort was focused on graphene $p-n$ junctions. ${ }^{8-13}$ In these devices, carriers tunnel through a sharp energy barrier induced with external local gate electrodes. Sophisticated nanofabrication techniques were employed to structure these local gates. For instance, the insulator layer was very thin, ${ }^{8,9,12}$ the local gate was separated from the graphene by an air gap, ${ }^{10,11}$ or the local gate was extremely narrow. ${ }^{13}$

Here, we argue that Zener-Klein tunneling can be observed in graphene with the most common device layout [undoped, four-point (4pt) configuration, and without any local gates] by simply measuring the $I-V$ at room temperature. On the basis of theoretical arguments, in graphene, the ZK current is expected to manifest itself with a superlinear current $I \propto V^{\alpha}$ with $\alpha=1.5$. The basic concepts can be understood with a semiclassical treatment which, in the case of ballistic transport, allows us to write an analytical expression for the $I-V$ 's as a function of the doping. Then, we study the role of defects with the "exact" (nonperturbative) nonequilibrium Green's function approach finding the counterintuitive result that charged impurities enhance the visibility of the ZK current. Finally, we report measurements showing that the $I-V$ 's at the Dirac point is indeed described by power laws, $I \propto V^{\alpha}$, with $\alpha$ ranging from 1 to 1.4. The exponent $\alpha$ is higher when the mobility is lower, consistently with theoretical predictions.

\section{THEORETICAL CONSIDERATIONS}

In graphene ZK tunneling leads to unusual $I-V$ 's as compared to those of semiconductors/insulators. Let us consider transport through a piece of a material and apply a voltage $-V$ between right $(\mathrm{R})$ and left $(\mathrm{L})$ sides. For a semiconductor with electronic gap $E_{g}$, ZK tunneling is possible only for $e V>E_{g}$, where $e>0$ is the electron charge (Fig. 1). On the contrary, in graphene the gap is zero and, thus, ZK tunneling is possible for arbitrarily small $V$.

More specifically, the two-dimensional electronic-band dispersion of graphene is a cone: $\epsilon= \pm \hbar v_{F} \sqrt{k_{\perp}^{2}+k^{2}}$, where $k$ $\left(k_{\perp}\right)$ is the wave-vector component parallel (perpendicular) to the current flow. During ballistic transport (in absence of scattering) $k_{\perp}$ is conserved. For a fixed $k_{\perp}$, the bands are hyperbolae with gap $\Delta=2 \hbar v_{F} k_{\perp}$ (Fig. 1). For any $V$, there are conducting channels for which the tunneling is possible (with $k_{\perp}$ such that $\Delta<V$ ). This results in a tunneling current $I \propto V^{3 / 2}$ (as shown below). By contrast, the ZK tunneling current in semiconductors vanishes exponentially at low $V$.

In graphene, within the Landauer approach, the current per unit of lateral length, $J$, is

$$
J=\frac{4 e}{h} \int \frac{d k_{\perp}}{2 \pi} \int_{\epsilon_{F}-e V}^{\epsilon_{F}} T\left(\epsilon, k_{\perp}, V\right) d \epsilon=\frac{4 e}{h} \int_{\epsilon_{F}-e V}^{\epsilon_{F}} \mathcal{T}(\epsilon, V) d \epsilon,
$$

where the factor 4 accounts for spin and valley degeneracies and the transmission $T\left(\epsilon, k_{\perp}, V\right)$ is the probability that an electron (with energy $\epsilon$ and perpendicular momentum $k_{\perp}$ ) is transmitted through the channel. We assume a uniform drop

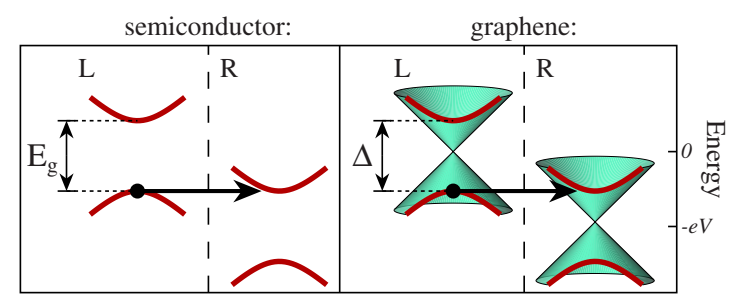

FIG. 1. (Color online) Bands of $\mathrm{L}$ and $\mathrm{R}$ contacts in a semiconductor and in graphene. The arrows represent the possible occurrence of Zener-Klein tunneling. 
of the electrostatic potential (not to be confused with the electrochemical potential, see Appendix C) along the current-flow direction, with constant electric field $V / l$, being $l$ the distance between the contacts. The validity of this approximation is discussed in Appendix C.

In the following we will show that, in the ballistic case (no defects), the transmission can be calculated with a semiclassical approach. A more reliable approach to determine the transmission is the one based on the nonequilibrium Green's function (NEGF) method. ${ }^{14}$ The NEGF approach is particularly well suited to study the role of defects, since it provides an exact (nonperturbative) atomistic treatment of disorder.

\section{A. Transport ballistic}

A semiclassical approach, based on the Wentzel-KramersBrillouin (WKB) approximation can be used to clarify the concept of Zener-Klein tunneling and to provide an analytical expression (valid only in the ballistic case) for the current-voltage curves as a function of the density of carriers. These expressions and their derivation are reported in Appendix A. The WKB method will be validated a posteriori since it reproduces very well the exact NEGF results.

Within a semiclassical treatment, the transmission $T^{\mathrm{WKB}}\left(\epsilon, k_{\perp}, V\right)$ can be equal to 1,0 , or to $T_{\mathrm{ZK}}=\exp \left[-\pi l \Delta^{2} /\right.$ $\left(4 \hbar v_{F} e V\right)$ ] (Refs. 4, 6, and 15) [see the example in Figs. 2(a) and 2(b)]. We call nontunneling current [Fig. 2(a)], the one associated with carriers that always remain in the same band $\pi$ or $\pi^{*}\left[T^{\mathrm{WKB}}=1\right.$, dark-shadowed (yellow) area in Fig. 2(a)]. We call "Zener-Klein" current, the one associated with carriers that tunnel from the $\pi$ to the $\pi^{*}$ band $\left[T^{\mathrm{WKB}}=T_{\mathrm{ZK}}\right.$, light-shadowed (cyan) area in Fig. 2(a)]. To verify whether this WKB approach is reliable, we also calculated the transmission with the NEGF method described in Appendix B. The approximated WKB transmission reproduces very well the more precise NEGF ones in all the relevant situations [see as an example the comparison reported in Fig. 2(b)].

In a graphene-based field-effect device, the density of the carriers $n$ can be varied by changing the gate voltage $V_{g} . n$ $=V_{g} C_{g} / e$, being $C_{g}$ the gate-channel capacitance. Figure 2(d) reports the current-voltage $(I-V)$ curves in the ballistic regime obtained with the semiclassical WKB approach [by letting $T=T^{\mathrm{WKB}}$ in Eq. (1), see Appendix A] and with the exact NEGF method for various dopings [we use $C_{g}=1.15$ $\times 10^{-4} \mathrm{~F} / \mathrm{m}^{2}$ (Ref. 16)]. The two methods give almost identical results. For zero doping $\left(V_{g}=0 \mathrm{~V}\right)$ there is no contribution from the nontunneling current, the current is entirely due to $\mathrm{ZK}$ tunneling, and the $I-V$ curve is superlinear $\left(I \propto V^{3 / 2}\right.$, see Appendix A). As soon as the system is doped (already for $V_{g}=5 \mathrm{~V}$ ) the $\mathrm{ZK}$ current is no more dominant (with respect to the nontunneling current, see Appendix A) and for small bias $(V<0.1 \mathrm{~V})$ the $I-V$ is essentially linear.

Do we expect the superlinear ZK current to be visible in actual devices? At first sight the answer is no for two reasons. First, in actual devices, the carrier concentration is never exactly zero. Indeed it has been observed ${ }^{17}$ that the presence of charge impurities induce a spatial fluctuation of the Fermi level with respect to the Dirac point. As a result, it
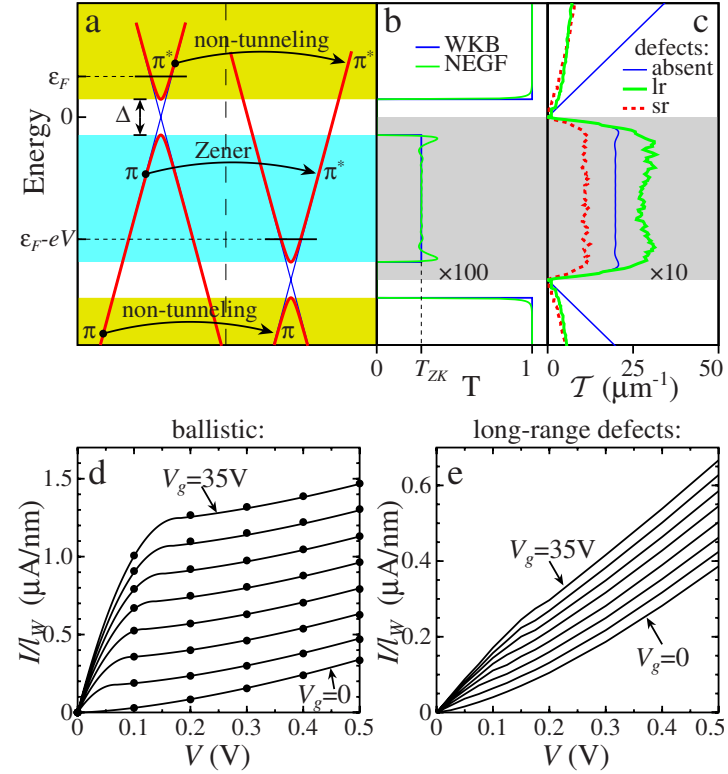

FIG. 2. (Color online) Electronic transport in graphene: theory. (a) Electronic bands of $\mathrm{L}$ and $\mathrm{R}$ contacts. The hyperbolae are the bands corresponding to a finite $k_{\perp}\left(\Delta=2 \hbar v_{F} k_{\perp}=22 \mathrm{meV}\right)$. In L and $\mathrm{R}$ contacts the bands are filled up to $\epsilon_{F}$ and $\epsilon_{F}-e V$, respectively, where $\epsilon_{F}>0\left(\epsilon_{F}<0\right)$ corresponds to electron (hole) doping. [(b) and (c)] Electronic transmissions $T$ and $\mathcal{T}$, defined as in Eq. (1), for $V=0.1 \mathrm{~V}$ and $l=1 \mu \mathrm{m}$. In the gray zone (the region corresponding to the Zener-Klein tunneling) $T$ and $\mathcal{T}$ are magnified. (b) Ballistic case calculated with NEGF or with the WKB approximation. (c) NEGF results in the ballistic case (no defects) or in the presence of lr or sr defects. [(d) and (e)] calculated current $I$ per unit of lateral length $l_{W}$ vs $V$ (the voltage applied between the electrodes) as a function of the gate voltage $\left(V_{g}\right) . V_{g}$ goes from 0 to $35 \mathrm{~V}$ with $5 \mathrm{~V}$ step, $l=1 \mu \mathrm{m}$. In the ballistic case (d), lines are approximated semiclassical results (i.e., the analytical curves from Appendix A), points are from the exact NEGF simulations.

is difficult to achieve the experimental condition where $\mathrm{ZK}$ tunneling is observable $\left[V_{g}=0\right.$ in Fig. 2(d) $]$. Second, the scattering of the carriers with optical phonons with energy $\hbar \omega$ $=0.15 \mathrm{eV}$ causes the current to saturate when increasing $V$ to high values ${ }^{16}$ (see also Ref. 18). This process occurs for $\mathrm{eV}$ $>\hbar \omega l / l_{e l}\left(l_{e l}\right.$ is the carrier elastic scattering length, due to defects) and is, thus, particularly relevant for high-quality high-mobility samples (with high $l_{e l}$ ). This saturation of the nontunneling current induces a sublinearity $\left(I \propto V^{\alpha}\right.$ with $\alpha$ $<1)$ which tends to cancel the superlinearity $(\alpha>1)$ of the ZK current, further masking it.

\section{B. Transport with defects}

The situation is possibly changed by the presence of defects. Actual devices are characterized by defects which scatter electrons elastically (that is conserving the energy). ${ }^{16}$ Elastic defects can be neutral point defects or charged Coulomb impurities ${ }^{19}$ outside the graphene plane (usually at a distance $\sim 1-2 \mathrm{~nm}) .^{20}$ Point defects affect the electrostatic potential seen by the carriers on a length scale smaller than the graphene unitary cell [short range (sr)] and, thus, the carriers cannot be described in terms of electronic bands. On 
the other hand, charged impurities modify the potential uniformly on a length scale much longer than the unit cell [long range (lr)] and the electronic bands are still a meaningful concept. The ZK current is expected to be more sensitive to short-range defects than to long-range ones. Indeed, the $\mathrm{ZK}$ current is determined by a transition between two bands whose relative energy is not affected by long-range defects. Moreover, long-range defects are expected to diminish the nontunneling current. Overall, one could wonder whether the presence of long-range defects can be used to suppress the nontunneling current and, thus, to make visible the $\mathrm{ZK}$ one.

To verify this hypothesis, we simulate disordered graphene within NEGF by considering both long- and shortrange elastic defects. We consider a tight-binding Hamiltonian and the NEGF equations are solved as in Ref. 21 (see Appendix B for further details). We consider a strip with lateral infinite width. Defect-induced disorder is simulated by changing the on-site potential by $V_{d}=0.1 \mathrm{eV}$ uniformly on trenches of atoms which are randomly distributed on along the channel. $V_{d}$ is uniform along the direction perpendicular to the channel (disorder does not break the lateral periodicity) for computational reasons. Long- (short-) range defects are simulated using trenches long $10 a_{0}\left(a_{0}\right)$, where $a_{0}$ is the graphene lattice parameter. $V_{d}=0.1 \mathrm{eV}$ is a realistic choice since it provides a low-bias conductivity in reasonable agreement with measurements (see Fig. 8 in Appendix B).

From NEGF simulations, the presence of long-range defects diminishes the nontunneling transmission [Fig. 2(c)] but, in general, does not reduce the $\mathrm{ZK}$ one. For $V=0.1 \mathrm{~V}$, long-range defects even increase the $\mathrm{ZK}$ transmission [Fig. 2(c)]. We checked that short-range defects diminish, as expected, both the nontunneling and the $\mathrm{ZK}$ transmission with respect to the ballistic case [Fig. 2(c)]. To see whether the relative increase in the $\mathrm{ZK}$ transmission with respect to the nontunneling one can lead to measurable effects, in Fig. 2(e) we show the theoretical $I-V$ curves in the presence of longrange defects. The superlinear behavior (the signature of the ZK current) is still visible at $V_{g}=0\left[I \propto V^{\alpha}\right.$ with $\alpha=1.4$ in Fig. 2(e)] and is also visible at finite $V_{g}$.

\section{COMPARISON WITH MEASUREMENTS}

We now turn our attention to measurements, carried out on single-layer graphene devices. Different devices were fabricated in a four-point configuration and have different mobilities $\mu$ ranging from 80 to $20000 \mathrm{~cm}^{2} \mathrm{~V}^{-1} \mathrm{~s}^{-1}$ (low mobility corresponds to a higher density of defects). See Appendix D for details on device fabrication and measurements. Figure 3(a) shows a typical set of $I-V$ characteristics for different $V_{g}$ applied on the backgate for a sample with a relatively modest mobility $\left(\mu=1700 \mathrm{~cm}^{2} \mathrm{~V}^{-1} \mathrm{~s}^{-1}\right)$. The $I-V$ is superlinear at the $V_{g}$ of the Dirac point, consistent with the above prediction of $\mathrm{ZK}$ tunneling. The superlinearity is better seen in a double-logarithmic scale plot [Fig. 3(c)] where the $I-V$ is reasonably well described by a power law $I \propto V^{\alpha}$ with $\alpha=1.3$. Both $\alpha$ and the current values are in a remarkable qualitative agreement with calculations given the simplicity of the model as can be seen by comparing Fig. 2(e)
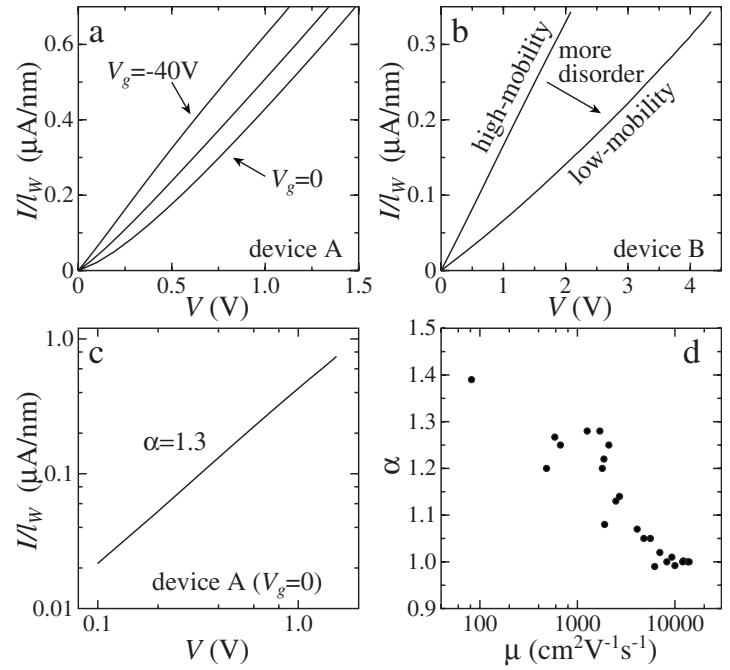

FIG. 3. Electronic transport in graphene: measurements. (a) Measured current $I$ per unit of lateral length $l_{W}$ vs $V$ in a lowmobility sample for different gate values $\left(V_{g}=0,-20,-40 \mathrm{~V}\right)$. $V_{g}$ has been shifted to $-14 \mathrm{~V}$ so that $V_{g}=0$ corresponds to the Dirac point. The length between the voltage electrodes $l=1.1 \mu \mathrm{m}$ and $l_{W}=1.1 \mu \mathrm{m}$. (b) Measured $I-V$ in a high-mobility sample, at the Dirac point, before and after the introduction of defects through electronic bombardment. $l=2.2 \mu \mathrm{m}$ and $l_{W}=550 \mathrm{~nm}$. (c) Doublelogarithmic scale plot of the $I-V$. (d) Exponent $\alpha$ as a function of mobility $\mu$ for different devices. $l$ varies from 0.9 to $5.9 \mu \mathrm{m}$ and $l_{W}$ from 70 to $1500 \mathrm{~nm}$.

$(l=1 \mu \mathrm{m})$ and Fig. 3(a) $(l=1.1 \mu \mathrm{m})$ for small $V_{g}$. Notice that calculations from Fig. 2 are obtained with a two-point (2pt) scheme. The comparison with the measurements of Fig. 3 (done in a four-point configuration) is, however, meaningful (see Appendix C). We remark that the calculations do not include scattering with optical phonons, which is relevant for $e V>\hbar \omega l / l_{e l}$.

We observe that the superlinearity vanishes for devices with high $\mu$. Figure 3(d) shows $\alpha$ (extracted at the Dirac point) as a function of the mobility $\mu$ of 22 different devices. As the mobility increases, $\alpha$ tends to 1 (corresponding to linear $I-V)$. In an additional experiment, we introduced defects in a high-mobility graphene device by bombarding it with $10 \mathrm{keV}$ electrons. From Fig. 3(b), before bombardment the mobility $\mu=7000 \mathrm{~cm}^{2} \mathrm{~V}^{-1} \mathrm{~s}^{-1}$ and the $I-V$ is linear with $\alpha=1.0$. After bombardment $\mu$ drops to $260 \mathrm{~cm}^{2} \mathrm{~V}^{-1} \mathrm{~s}^{-1}$ and the $I-V$ becomes superlinear $(\alpha=1.2)$. These observations are consistent with the previous discussion that in high-mobility samples the ZK superlinearity is masked by the nontunneling current. Namely, the reduction in disorder increases the contribution of the nontunneling current with respect to the $\mathrm{ZK}$ one and, also, favors the nontunneling current saturation (due to scattering with optical phonons, ${ }^{16,18}$ see Appendix E for further discussions). More elaborated models (e.g., with a more realistic description of impurities, including electronphonon scattering and possible self-heating effects) are required to reach a quantitative agreement between theory and measurements.

We now discuss other mechanisms that could lead to superlinear $I-V$ 's. It could be related to the physics occurring at 
tunnel barriers (such as the Luttinger liquidlike behavior in nanotubes ${ }^{22,23}$ or the breakdown of insulating barriers). However, measurements are done on high-quality devices in a four-point configuration, which makes the presence of tunnel barriers unlikely. Superlinear $I-V$ 's could also be attributed to quantum effects, such as weak localization or electronelectron interaction, but these effects should be negligible since the applied current is large, heating the graphene layer to several hundreds of celsius. ${ }^{24}$ Overall, Zener-Klein tunneling remains the most plausible mechanism to explain our measurements.

We finally stress that previous observations of Klein tunneling ${ }^{10,12,13}$ in graphene were done using very different device setups. In Refs. 10, 12, and 13, the carriers tunnel from conduction to valence bands in a $p-n$ junction. In these nanostructured devices, the ZK tunneling is observed thanks to a configuration which allows to eliminate the nontunneling current and thanks to the intense electric field at the $p-n$ junction $\left(\sim 10^{-3} \mathrm{eV} / \AA\right.$, see supplementary information of Refs. 9 and 13). In our devices, which are not $p$ - $n$ junctions, the nontunneling (intraband) current is present (this current can mask the $\mathrm{ZK}$ tunneling one) and the electric field $\left(\sim 10^{-5} \mathrm{eV} / \AA\right)$ is substantially weaker. Despite these unfavorable conditions, it is possible to probe the Zener-Klein effect.

\section{CONCLUSIONS}

Concluding, measurements and calculations show, consistently, that the $I-V$ 's of graphene devices become superlinear in the presence of disorder (in low-mobility samples). The superlinearity is attributed to Zener-Klein tunneling (tunneling between different energy bands, from $\pi$ to $\pi^{*}$ ). In highmobility (high-quality) graphene samples, the superlinearity is masked by the contribution of the nontunneling current (due to carriers always remaining the same band). In lowmobility samples, the Zener-Klein tunneling current is visible because the higher density of defects decreases (filters) the nontunneling current but, surprisingly, does not degrade (to the same extent) the tunneling one.

Note added in proof: Recently we became aware of two papers predicting a super-linear behavior at high bias in graphene due to interband tunneling. ${ }^{25,26}$

\section{ACKNOWLEDGMENTS}

We thank G. Stoltz. The research was supported by an EURYI Grant, an EU under Grant No. FP6-IST-021285-2, the ANR PNANO-ACCATTONE and IDRIS (Orsay).

\section{APPENDIX A: BALLISTIC REGIME WITHIN THE WKB APPROXIMATION}

Here, we derive a set of analytical formula for the currentvoltage curves as a function of the carrier concentration in the ballistic regime (neither defects nor phonon scattering), within a semiclassical approach.

We consider a graphene sample and apply a voltage $V$ between the two contacts. We assume a linear drop of the electrostatic potential along a channel of length $l$, corre-

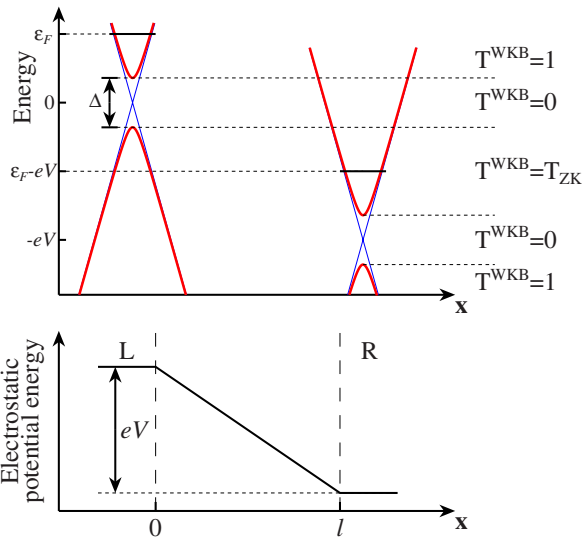

FIG. 4. (Color online) Schematic band structure and transition probabilities of a graphene junction.

sponding to a constant electric field $V / l$ directed along $x$. In a semiclassical trajectory, the electron wave vector along the direction perpendicular to the field is conserved, i.e., $k_{\perp}$ $=$ constant $=\Delta /\left(2 \hbar v_{F}\right)$, where $v_{F}$ is the graphene Fermi velocity $\left(\hbar v_{F} \simeq 6.4 \mathrm{eV} \AA\right.$ ). Figure 4 indicates the graphene band structure of the two contacts along a line with $k_{\perp}=$ constant. For the energies for which the two conduction bands (or the two valence bands) of the two contact overlap, the transmission probability $T^{\mathrm{WKB}}=1$. For energies where there is no overlap at all between a left constant band and a right constant band, $T^{\mathrm{WKB}}=0$. Finally, for energies where a valence (conduction) band of the left contact overlaps with the conduction (valence) band of the right contact $T^{\mathrm{WKB}}=T_{\mathrm{ZK}}$ and can be computed within the WKB theory. ${ }^{15}$ The WKB momentum $k_{x}$, at a position $x$ in the channel, is given by

$$
k_{x}=\sqrt{\left(\frac{e V x}{\hbar v_{F} l}\right)^{2}-k_{\perp}^{2}} .
$$

The transmission is obtained after an integration on the classically forbidden region

$$
\begin{aligned}
T_{\text {ZK }} & =\exp \left[-2 \operatorname{Im} \int_{-\infty}^{\infty} d x k_{x}\right] \\
& =\exp \left[-\frac{2 k_{\perp}^{2} \hbar v_{F} l}{e V} \int_{-1}^{1} d s \sqrt{1-s^{2}}\right] \\
& =\exp \left[-\frac{\pi k_{\perp}^{2} \hbar v_{F} l}{e V}\right]
\end{aligned}
$$

The current is obtained by letting $T=T^{\mathrm{WKB}}$ in Eq. (1). Let us write the total current per unit of lateral length as $J=J_{n t}$ $+J_{Z}$, where $J_{n t}$ and $J_{Z}$ are the nontunneling and ZK currents, For $\epsilon_{F}=0$,

$$
\begin{gathered}
J_{n t}=0, \\
J_{Z}=2 J_{0} \mathcal{F}\left(\kappa_{0}\right),
\end{gathered}
$$

where $J_{0}=V /\left(\pi^{2} R_{0} l\right)$ with $R_{0}=\pi \hbar / e^{2}=12.9064 \mathrm{k} \Omega$ the quantum of resistance. $\kappa_{0}=\pi e V l /\left(4 \hbar v_{F}\right)=1.21 V l /(\mathrm{nm} \mathrm{V})$ is adimensional, and $\mathcal{F}(x)=\sqrt{\pi x} \operatorname{erf}(\sqrt{x})+e^{-x}-1$. For typical 


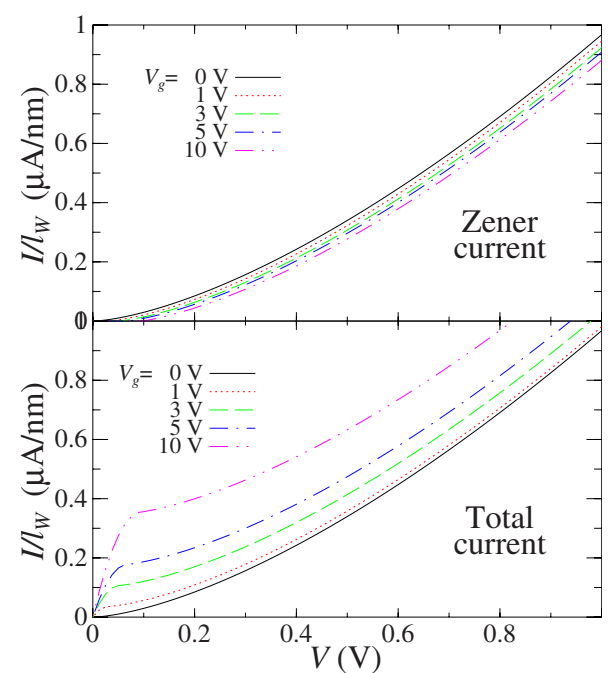

FIG. 5. (Color online) Zener current $\left(J_{Z}\right)$ and total one $\left(J_{Z}\right.$ $+J_{n t}$ ) from Eqs. (A3) and (A4) for various gate voltages $V_{g}$. The length $l=1 \mu \mathrm{m}$.

lengths of a few micron, if $V \gg 1 \mathrm{mV}, \kappa_{0} \gg 1$ and $J_{Z}$ $\simeq 2 J_{0} \sqrt{\pi \kappa_{0}} \propto V^{3 / 2}$. At these conditions the ballistic Zener conductance is

$$
\sigma_{b Z}=J_{Z} l / V \simeq 2 \sqrt{\pi \kappa_{0}} /\left(\pi^{2} / R_{0}\right)=0.398 / R_{0} \sqrt{V l /(\mathrm{nm} \times \mathrm{V})} .
$$

For $\epsilon_{F} \neq 0$ we distinguish three cases

$$
\begin{gathered}
e V<\epsilon_{F} \quad J_{n t}=J_{1}\left(2-e V / \epsilon_{F}\right) e V / \epsilon_{F}, \\
J_{Z}=0, \\
\epsilon_{F}<e V<2 \epsilon_{F} \quad J_{n t}=J_{1}, \\
J_{Z}=J_{0} \mathcal{F}\left[\kappa_{1}\left(e V / \epsilon_{F}-1\right)^{2}\right], \\
2 \epsilon_{F}<e V \quad J_{n t}=J_{1}, \\
J_{Z}=J_{0}\left[2 \mathcal{F}\left(\kappa_{0}\right)-\mathcal{F}\left(\kappa_{1}\right)\right],
\end{gathered}
$$

where $J_{1}=e v_{F} n / \pi$, being $n=\epsilon_{F}^{2} / \pi /\left(\hbar v_{F}\right)^{2}$ the electron concentration (number of carriers per surface unit cell), and $\kappa_{1}$ $=J_{1} / J_{0}$ is adimensional. Note that the calculated saturation of the nontunneling current at $e V=\epsilon_{F}$ in the ballistic regime $\left[J_{n t}=J_{1}\right.$, see Fig. 2(d) $]$ should not be confused with the saturation due to optical-phonon scattering described in Refs. 16 and 18. For $V_{g}=0$, the current is entirely due to Zener tunneling $\left(J_{n t}=0\right)$. By increasing $V_{g}$, the Zener current decreases (Fig. 5 upper panel) while the total one increases (Fig. 5 lower panel). Thus, already at $V_{g}=5 \mathrm{~V}$ the nontunneling current dominates over the Zener one. Note that, for simplicity, we have neglected the electronic temperature. We checked numerically that such an approximation is valid for voltages $e V \gg K_{B} T$, as expected.

Equations (A3) and (A4) can be easily derived as follows. First we consider the Zener current. For $\epsilon_{F}=0$, the Zener tunneling operates, for a given $k_{\perp}$ in an energy window of width $e V-\Delta=e V-2 \hbar v_{F} k_{\perp}$ (see Fig. 4). So, the Zener tunnel- ing occurs only if $k_{\perp}<e V /\left(2 \hbar v_{F}\right)$. The current (per lateral unit length), $J_{Z}$ according to a Landauer formalism is

$$
\begin{aligned}
J_{Z} & =\frac{4 e}{2 \pi \hbar} \int_{-k_{m}}^{k_{m}} \frac{d k_{\perp}}{2 \pi} \int_{0}^{k_{n}} d k_{x} \frac{d \epsilon_{\mathbf{k}, \pi^{*}}}{d k_{x}} e^{-a k_{\perp}^{2}} \\
& =\frac{4 e}{\pi \hbar} \int_{0}^{k_{m}} \frac{d k_{\perp}}{2 \pi}(e V-\Delta) e^{-a k_{\perp}^{2}} \\
& =\frac{4 e v_{F}}{\pi^{2}} \int_{0}^{k_{m}} d k_{\perp}\left(k_{m}-\left|k_{\perp}\right|\right) e^{-a k_{\perp}^{2}} \\
& =2 \frac{e v_{F}}{\pi^{2} a} 2 a k_{m}^{2} \int_{0}^{1} d s(1-s) e^{-a k_{m}^{2} s^{2}} \\
& =2 J_{0} \mathcal{F}\left(\kappa_{0}\right),
\end{aligned}
$$

where 4 count spin, and valley degeneracy, and

$$
\begin{gathered}
k_{m}=\frac{e V}{2 \hbar v_{F}}, \\
k_{n}=\sqrt{\left(\frac{e V}{\hbar v_{F}}\right)^{2}-k_{\perp}^{2},} \\
\epsilon_{\mathbf{k}, \pi^{*}}=\hbar v_{F}\left(k_{x}^{2}+k_{\perp}^{2}\right)^{1 / 2}, \\
a=\frac{\pi \hbar v_{F} l}{e V}, \\
J_{0}=\frac{e v_{F}}{\pi^{2} a}=\frac{e^{2} V}{\pi^{3} \hbar l}, \\
\kappa_{0}=a k_{m}^{2}=\frac{\pi e V l}{4 \hbar v_{F}}, \\
\mathcal{F}(\kappa)=2 \kappa \int_{0}^{1}(1-s) e^{-\kappa s^{2}} d s=\sqrt{\pi \kappa} \operatorname{erf}(\sqrt{\kappa})+e^{-\kappa}-1 .
\end{gathered}
$$

Now, we consider the Zener current for an arbitrary $\epsilon_{F} \geq 0$. For $e V<\epsilon_{F}, J_{Z}=0$. When $\epsilon_{F}<e V<2 \epsilon_{F}$

$$
\begin{aligned}
J_{Z} & =\frac{4 e}{\pi \hbar} \int_{0}^{k_{l}} \frac{d k_{\perp}}{2 \pi}\left(e V-\frac{\Delta}{2}-\epsilon_{F}\right) e^{-a k_{\perp}^{2}} \\
& =\frac{2 e v_{F}}{\pi^{2}} \int_{0}^{k_{l}} d k_{\perp}\left(k_{l}-k_{\perp}\right) e^{-a k_{\perp}^{2}} \\
& =\frac{e v_{F}}{\pi^{2} a} 2 a k_{l}^{2} \int_{0}^{1} d s(1-s) e^{-a k_{l}^{2} s^{2}} \\
& =J_{0} \mathcal{F}\left[\kappa_{1}\left(e V / \epsilon_{F}-1\right)^{2}\right],
\end{aligned}
$$

where

$$
k_{l}=\frac{e V-\epsilon_{F}}{\hbar v_{F}},
$$




$$
\begin{gathered}
k_{F}=\frac{\epsilon_{F}}{\hbar v_{F}}, \\
\kappa_{1}=\frac{\pi l \epsilon_{F}^{2}}{\hbar v_{F} e V}=a k_{F}^{2}=\frac{J_{1}}{J_{0}} .
\end{gathered}
$$

For $e V>2 \epsilon_{F}$ the Zener current becomes

$$
\begin{aligned}
J_{Z}= & \frac{4 e}{\pi \hbar} \int_{k_{F}}^{k_{m}} \frac{d k_{\perp}}{2 \pi}(e V-\Delta) e^{-a k_{\perp}^{2}} \\
& +\frac{4 e}{\pi \hbar} \int_{0}^{k_{F}} \frac{d k_{\perp}}{2 \pi}\left(e V-\frac{\Delta}{2}-\epsilon_{F}\right) e^{-a k_{\perp}^{2}} \\
= & \frac{4 e v_{F}}{\pi^{2}} \int_{0}^{k_{m}} d k_{\perp}\left(k_{m}-k_{\perp}\right) e^{-a k_{\perp}^{2}} \\
& -\frac{2 e v_{F}}{\pi^{2}} \int_{0}^{k_{F}} d k_{\perp}\left(k_{F}-k_{\perp}\right) e^{-a k_{\perp}^{2}} \\
= & J_{0}\left[2 \mathcal{F}\left(\kappa_{0}\right)-\mathcal{F}\left(\kappa_{1}\right)\right] .
\end{aligned}
$$

Now, we consider the nontunneling current, an arbitrary $\epsilon_{F}$ $\geq 0$. The nontunneling current operates, for a given $k_{\perp}$ in an energy window between $\epsilon_{F}$ and $\max \left(\hbar v_{F} k_{\perp}, e V-\epsilon_{F}\right)$ (see Fig. 4). Thus, the current (per lateral unit length), $J_{n t}$ according to a Landauer formalism is

$$
\begin{aligned}
J_{n t} & =\frac{8 e}{2 \pi \hbar} \int_{0}^{\epsilon_{F} / \hbar v_{F}} \frac{d k_{\perp}}{2 \pi}\left[\epsilon_{F}-\max \left(\hbar v_{F} k_{\perp}, e V-\epsilon_{F}\right)\right] \\
& =\frac{2 e}{\pi^{2} \hbar^{2} v_{F}} \int_{0}^{\epsilon_{F}} d \epsilon\left[\epsilon_{F}-\max \left(\epsilon, \epsilon_{F}-e V\right)\right] .
\end{aligned}
$$

If $e V>\epsilon_{F}$ the current saturates to $J_{n t}=J_{1}$ with

$$
J_{1}=\frac{e \epsilon_{F}^{2}}{\pi^{2} \hbar^{2} v_{F}}=\frac{\epsilon_{F}^{2}}{\pi R_{0} e \hbar v_{F}}=\frac{e v_{F} n}{\pi},
$$

where $R_{0}=\pi \hbar / e^{2}$ and $n=\epsilon_{F}^{2} /\left[\pi\left(\hbar v_{F}\right)^{2}\right]$ is the numbers of electron per unit area. If $e V<\epsilon_{F}$,

$$
\begin{aligned}
J_{n t} & =\frac{2 e}{\pi^{2} \hbar^{2} v_{F}}\left[e V\left(\epsilon_{F}-e V\right)+\int_{\epsilon_{F}-e V}^{\epsilon_{F}} d \epsilon\left(\epsilon_{F}-\epsilon\right)\right] \\
& =J_{1}\left(2-e V / \epsilon_{F}\right) e V / \epsilon_{F} .
\end{aligned}
$$

\section{APPENDIX B: NEGF CALCULATIONS}

Here we describe the NEGF approach ${ }^{14}$ which is used to compute the transmission of graphene in presence of disorder. We use an orthogonal tight-binding Hamiltonian with one $p_{z}$ orbital per carbon atom, and nearest-neighbor hopping $t=-3 \mathrm{eV}\left(v_{F}=10^{8} \mathrm{~cm} / \mathrm{s}\right)$. The external electrical field and the impurities affect only the on-site potential, the hopping and overlap are unchanged. The conduction properties per lateral unit length are independent of the lateral edge termination. In our calculation we consider graphene samples infinitely wide (in the $\perp$ direction), where both the leads and channel are made out of graphene. The left (right) leads is

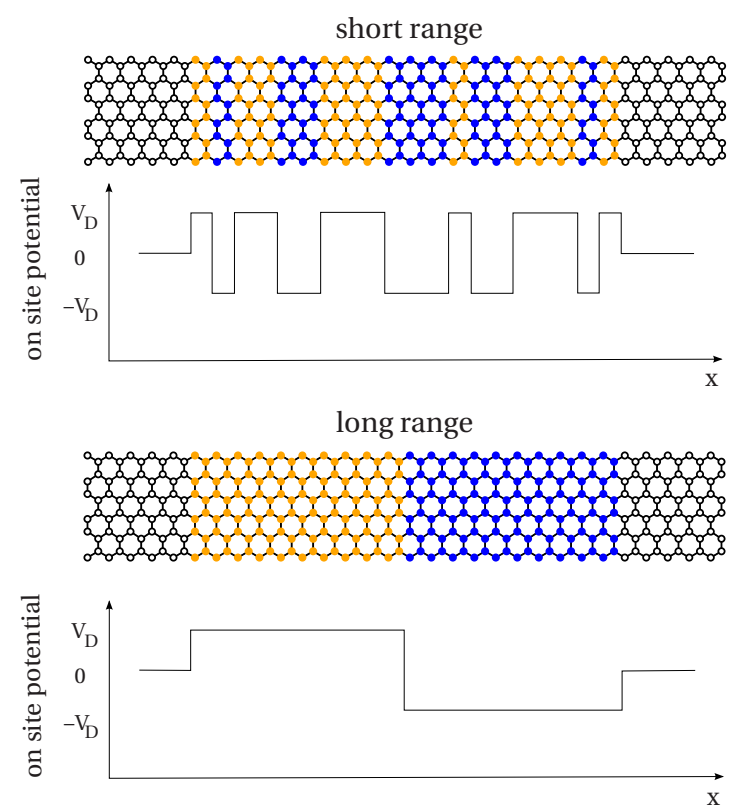

FIG. 6. (Color online) Scheme of short- and long-range disorder. The dark and the light (red and blue) dots correspond to an on-site potential $+V_{d}$ and $-V_{d}$. The perfect leads are shown by the white dots where the on-site potential vanishes.

defect free, and semi-infinite in the $-x(+x)$ direction. The electrostatic potential is constant within the two leads. The graphene channel between the two leads has a length (in the $x$ direction) of $\sim 1 \mu \mathrm{m}$, which is comparable with the distance between contacts in the experimental samples. The channel is placed in a constant electric field along $x$. The graphene lattice is oriented as shown in Fig. 6, i.e., we consider infinitely wide zigzag ribbons. In real samples the disorder scatters the electrons in both the directions along and perpendicular to the channel. Within our approach, such a disorder could be modeled using supercells with a periodicity $W$ along $\perp$. The width $W$ should allow lateral $(\perp)$ scattering within the Fermi surface, i.e., $W \gg 2 \pi / k_{F}$, where the Fermi wave-vector $k_{F}=\epsilon_{F} /\left(\hbar v_{F}\right), \epsilon_{F}=\hbar v_{F} \sqrt{\pi \eta V_{g}}$, and, in the present experimental setup, $\eta=7 \times 210^{10} \mathrm{~cm}^{-2} \mathrm{~V}^{-1}$. As an example, for a value of the gate potential $V_{g}=5 \mathrm{~V}, W$ $\gg 2 \pi / \sqrt{\pi \eta V_{g}} \simeq 0.1 \mu \mathrm{m}$. The present algorithms for the computation of the transmission within a Green's function approach requires a computational time that scales as $W^{3} L$, where $L$ is the channel length and $W$ is the channel width. ${ }^{21}$ Since with the available computational resources it is impossible to deal with systems with both length and lateral sizes on the order of micron, we neglect the lateral scattering and we consider a one-dimensional disorder along the $x$ direction (the disorder is taken to be invariant in the $\perp$ direction). In this case the lateral periodic unit cell is four atoms wide, see Fig. 7 and the transverse momentum $k_{\perp}$ is a good quantum number. For each $k_{\perp}$, the transmission can be computed on a narrow graphene sample with four atoms in the $\perp$ direction as shown in Fig. 7. The transmission is then averaged over $k_{\perp}$.

The two disorder models, henceforth called short- and long-range disorder, used in this study are shown in Fig. 6. In 


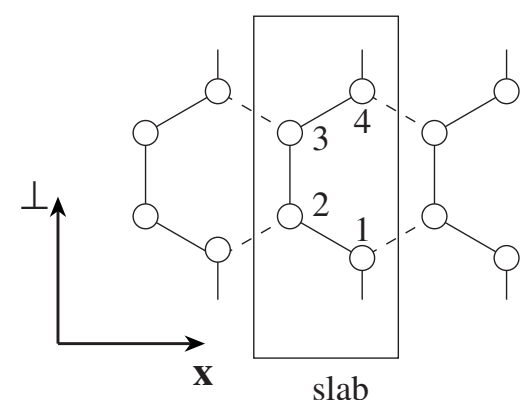

FIG. 7. Scheme of the slab used in the NEGF calculations. For a given transverse wave vector, the transmission is computed on a narrow graphene sample with four atoms in the $\perp$ direction.

the following, a slab is defined as shown in Fig. 7. For the short- (long-) range disorder, the on-site potential in one (ten sequent) slab(s) is a random variable equal to $+V_{d}$ with probability 0.5 and $+V_{d}$ with probability 0.5 .

The channel Green's function is

$$
G=\left(E+\imath \delta-\mathcal{H}_{D}-\Sigma_{\mathrm{L}}-\Sigma_{\mathrm{R}}\right)^{-1},
$$

where $\delta=10^{-8} \mathrm{eV}$ is a small positive smearing parameter and $\Sigma_{L}\left(\Sigma_{R}\right)$ is the self-energies due to the left (right) lead. The self-energies $\Sigma_{L}$ and $\Sigma_{R}$ are computed using the decimation algorithm. The transmission is computed using the algorithm described in Ref. 21 in the context of phonons elastic scattering. The transmission is derived using

$$
T_{k_{\perp}}=\operatorname{Tr}\left(\Gamma_{\mathrm{L}} G_{D} \Gamma_{\mathrm{R}} G_{D}^{\dagger}\right),
$$

where the subscript $k_{\perp}$ recalls that, at this point, only one transverse wave vector is considered, and

$$
\Gamma_{\mathrm{L} / \mathrm{R}}=\imath\left(\Sigma_{\mathrm{L} / \mathrm{R}}-\Sigma_{\mathrm{L} / \mathrm{R}}^{\dagger}\right)
$$

The total transmission is obtained by summing over $M$ transverse wave vectors. The transmission per unit width is

$$
\mathcal{T}=\frac{1}{M W} \sum_{j=0}^{M-1} T_{k_{\perp}=j 2 \pi /(M W)},
$$

where $W=\sqrt{3} a_{0}$ and $a_{0}=2.46 \AA$ is the graphene lattice constant.

In the calculation, for energies corresponding to Zener transmission, we use $M W=3.2 \mu \mathrm{m}$ and, for the normal transmission, we use $M W=0.8 \mu \mathrm{m}$. The length of the graphene channel is $0.98 \mu \mathrm{m}$ which corresponds to 4000 slabs. The transmission in Fig. 2 of the paper is averaged over 200 disorder realizations. The transmissions used for the current are averaged 100 times since the integration over the energies smooths the results.

We used a value of $V_{d}=0.1 \mathrm{~V}$ to describe the disorder. With such a choice the computed conductivities (at low field) with short- and long-range disorder are similar to those measured experimentally, see Fig. 8.

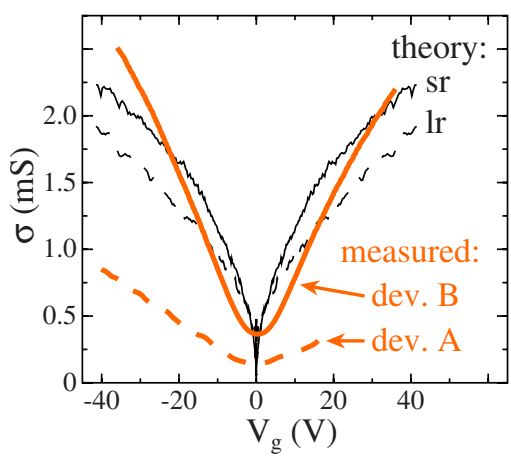

FIG. 8. (Color online) Conductivity $(\sigma)$ vs gate voltage $\left(V_{g}\right)$ of graphene. Measurements are done on two devices (dev. A and B) described in the text. Calculations are done using NEGF $\left(V_{d}\right.$ $=0.1 \mathrm{~V})$ with sr and $1 \mathrm{r}$ defects.

\section{APPENDIX C: ELECTROCHEMICAL POTENTIAL: FOUR-POINT VS TWO-POINT MEASUREMENTS}

It is important to distinguish the electrostatic potential from the electrochemical potential. ${ }^{14}$ The electrostatic potential is determined from the charge distribution of the system. In general, it has to be determined self-consistently from the local charge distribution by solving the Poisson equation. In the present specific case, we assume that the electrostatic potential is entirely determined by the charge accumulated in the current contacts [A and D in Fig. 11(b)] and, for simplicity, we also assume that the electrostatic potential decays linearly. The electrochemical potential is a local quasi-Fermi energy (see Ref. 14 for a discussion) which corresponds to the quantity measured by the voltage contacts $[\mathrm{B}$ and $\mathrm{C}$ in Fig. 11(b)] in a multiterminal device. We now show how the electrochemical potential can be computed.

We now consider just one of the two equivalent Dirac cones. For a given $k_{\perp}$ and energy $\epsilon$, the wave functions inside the contact regions $(x<0$ and $x>l$ in the lower panel of Fig. 4) can be classified as $\phi_{k_{\perp}}^{+}(x)$ and $\phi_{k_{\perp}}^{-}(x)$ for $x<0$ (in the left contact) and $\widetilde{\phi}_{k_{\perp} \epsilon}^{+}(x)$ and $\widetilde{\phi}_{k_{\perp} \epsilon}^{-}(x)$ for $x>l$ (in the right contact). The superscripts + and - indicate wave functions moving toward the right and the left directions, respectively. The $\phi$ functions are normalized to one on the graphene unit cell. We now consider the usual definition of left/right (L/R) scattering states ${ }^{14}$ that are the wave functions $\psi_{k_{\perp}}^{\mathrm{L}} \epsilon(x)$ and $\psi_{k_{\perp}}^{\mathrm{R}}(x)$ defined by the conditions at the border:

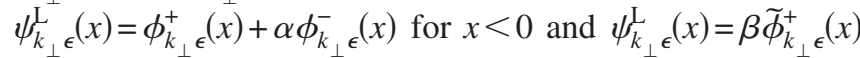
for $x>l ; \psi_{k_{\perp} \epsilon}^{\mathrm{R}}(x)=\widetilde{\phi}_{k_{\perp}}^{-} \epsilon^{-}(x)+\alpha^{\prime} \widetilde{\phi}_{k_{\perp}}^{+} \epsilon^{\prime}(x)$ for $x>l$ and $\psi_{k_{\perp}}^{R^{+}}(x)$ $=\beta^{\prime} \phi_{k_{\perp}}^{-} \epsilon^{\prime}(x)$ for $x<0 . \alpha, \beta, \alpha^{\prime}$, and $\beta^{\prime}$ are to be determined by imposing that the scattering states are eigenstates of the Hamiltonian. $\psi^{\mathrm{L}}$ and $\psi^{\mathrm{R}}$ describe an electronic state coming from left and right contacts, respectively.

We now consider a metallic probe in a point $x$ of the graphene sample. $\mu(x)$ is the electrochemical potential of the probe. Assuming that the transmission probability between the probe and graphene does not depend on $\epsilon$ and $k_{\perp}$ and that the density of states of the probe is constant, the current $J_{g p}$ between graphene and the probe is 


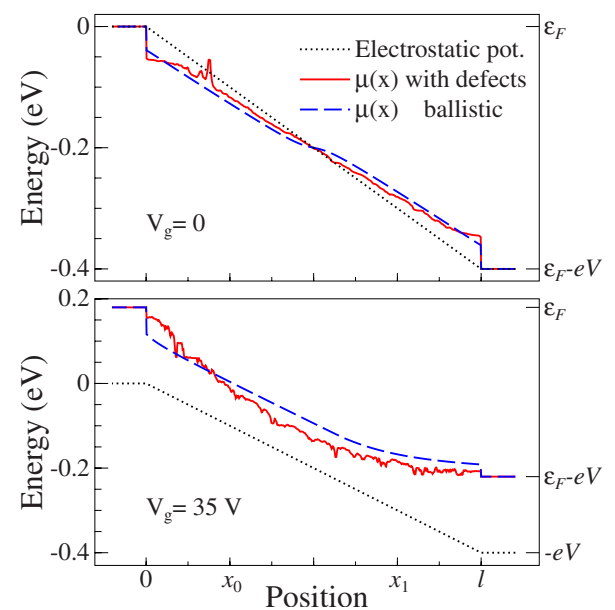

FIG. 9. (Color online) Calculated electrostatic potential and electrochemical potential, $\mu(x)$, as a function of the position $x$ along a graphene channel. Calculations are done in the ballistic case (no defects) or in the presence of long-range defects for two different gate voltages $V_{g}$. The length of the channel is $l=2 \mu \mathrm{m}$ and the applied voltage $V=0.4 \mathrm{~V}$.

$$
\begin{gathered}
J_{g p}(x)=C \int d k_{\perp} \int d \epsilon, \\
g_{\mathrm{L}}\left(k_{\perp}, \epsilon\right)\left|\psi_{k_{\perp}}^{\mathrm{L}}(x)\right|^{2}\left\{f\left[\epsilon-\epsilon_{F}^{\mathrm{L}}\right]-f[\epsilon-\mu(x)]\right\}+g_{\mathrm{R}}\left(k_{\perp}, \epsilon\right) \\
\times\left|\psi_{k_{\perp}}^{\mathrm{R}}(x)\right|^{2}\left\{f\left[\epsilon-\epsilon_{F}^{\mathrm{R}}\right]-f[\epsilon-\mu(x)]\right\},
\end{gathered}
$$

where $C$ is a constant, $g_{\mathrm{L}}\left(k_{\perp}, \epsilon\right)$ and $g_{\mathrm{R}}\left(k_{\perp}, \epsilon\right)$ are the densities of states at that $\epsilon$ and $k_{\perp}$ in left and right contacts, and $f(\epsilon)$ is the Fermi occupation function (we assume zero temperature). $\epsilon_{F}^{\mathrm{L}}=\epsilon_{F}$ and $\epsilon_{F}^{\mathrm{R}}=\epsilon_{F}-e V$ are the Fermi levels in left and right contacts. In four-point measurement (Fig. 11), the current between the voltage contacts [B and C in Fig. 11(b)] and the graphene channel is zero and the electrochemical potential of the voltage contacts coincides with the one of graphene in that point $x$. Thus, the $\mu(x)$ such that $J_{g p}(x)=0$ determines the electrochemical potential in the point $x$ of the graphene sample as it is measured in a four-probe measurement.

In Fig. 9, we show some examples of the electrostatic potential and of the electrochemical potential $\mu(x)$ (determined with the above procedure) in the ballistic case and in the presence of long-range defects for two different gate voltages $V_{g}$. One should notice the discontinuity of $\mu(x)$ at $x=0$ and $x=l$. These jumps correspond to the contact resistance at the current electrodes. We remark that, in all the cases, the jump is small compared to overall potential drop. This implies that the contact resistance in the simulations is small compared to that of the channel. This is true for $V_{g}$ $=0$, where the current is only due to Zener tunneling, and also for $V_{g}=35 \mathrm{~V}$, where the nontunneling current is dominating. Moreover, this is true in the presence of defects and also in the ballistic case. We also remark that the drop of $\mu(x)$ is quite uniform along the channel and that, in the central region, it has a slope similar to the one of the electrostatic potential.
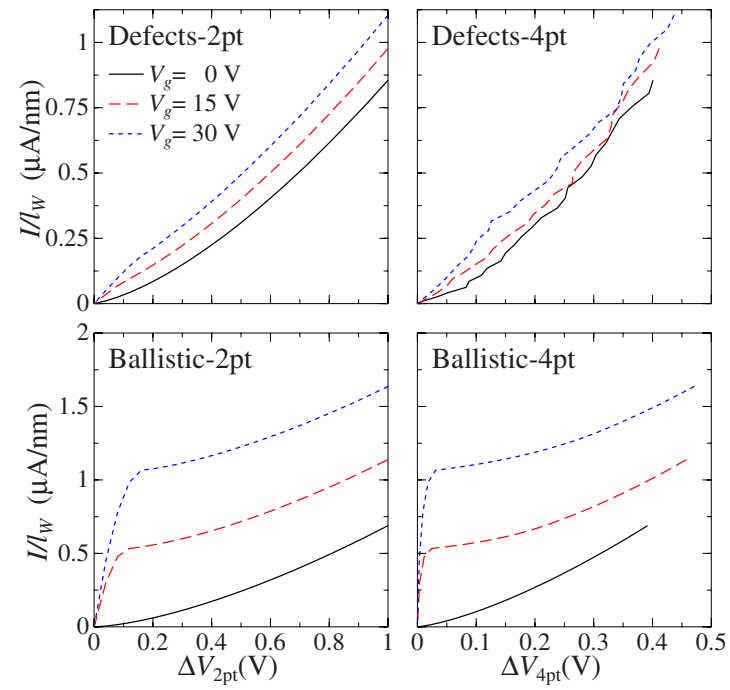

FIG. 10. (Color online) Comparison of the $I-V$ characteristics calculated in a $2 \mathrm{pt}$ configuration and in a $4 \mathrm{pt}$ one, in the ballistic case (no defects) or in the presence of long-range defects for various gate voltages $V_{g} . \Delta V_{2 \mathrm{pt}}$ is the drop of the electrostatic potential along the length of the channel, $l=2 \mu \mathrm{m} . \Delta V_{4 \mathrm{pt}}$ is $\Delta \mu$ along a $l / 2$ distance in the middle of the channel (see the text).

In the main text of the paper, we are comparing experimental $I-V$ curves measured in a four-point configuration with theoretical curves which correspond to a two-point configuration. We now show why this comparison is meaningful. In a four-point measurement the relevant voltage is the drop of the electrochemical potential between the two voltage contacts [B and $\mathrm{C}$ in Fig. 11(b)]. In a two-point configuration, the relevant voltage is the drop of the potential between the two current contacts [A and D in Fig. 11(b)]. In order to simulate four-point measurements in a configuration similar to the experimental one, in Fig. 10 we plot the current vs the drop to the electrochemical potential along a $l / 2(l$ is the channel length) distance in the middle of the channel $(\Delta \mu$ between $x_{0}$ and $x_{1}$ in Fig. 9, $\Delta V_{4 \mathrm{pt}}$ in Fig. 10). Figure 10 also report the current as a function of the drop of the electrostatic potential along the length of the channel ( $V$ in Fig. $2, \Delta V_{2 \mathrm{pt}}$ in Fig. 10). By comparing the curves obtained with the $4 \mathrm{pt}$ configuration with the $2 \mathrm{pt}$ ones, in all the cases, the overall shape and behavior is very similar apart from a rescale of the voltage axis. This is due to the facts that the contact resistance is small and that the drop of the electrochemical potential is quite uniform along the channel (Fig. 9), as discussed in the previous paragraph. This justifies, a posteriori, the use of a two-point configuration in the theoretical model discussed in the main text of the paper.

\section{APPENDIX D: SAMPLE FABRICATION AND EXPERIMENTAL SETUP}

Graphene devices are fabricated as in Ref. 16 by means of standard nanofabrication techniques. Graphene flakes are obtained by mechanical exfoliation of kish graphite (Toshiba Ceramics) by adhesive tapes on highly doped silicon wafers coated with a 280-nm-thick thermal silicon-oxide layer. 

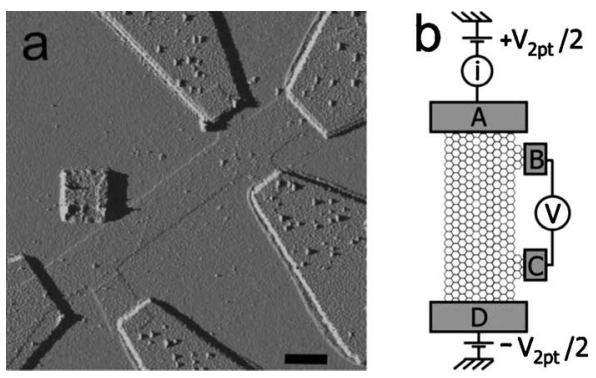

FIG. 11. (a) Atomic force microscopy image of a graphene device. The scale bar is $1 \mu \mathrm{m}$. (b) Schematic of the setup for the four-point measurement. The device is symmetrically voltage biased.

Single layers are identified with Raman spectroscopy and/or by measuring the reflected light intensity using a camera mounted on an optical microscope. Graphene layers are patterned in a Hall-bar configuration using $\mathrm{O}_{2}$ plasma etching. $\mathrm{Cr} / \mathrm{Au}$ electrodes are fabricated on top of the samples using electron-beam lithography followed by a lift-off in acetone and dicholoroethane. Devices are cleaned using thermal annealing at $350 \mathrm{C}$ in $\mathrm{H}_{2} / \mathrm{Ar}$ for a few hours and the currentinduced cleaning technique in vacuum. ${ }^{27}$ Figure 11(a) shows an atomic force microscopy image of a device at the end of the fabrication process.

We employ a four-point configuration to avoid the contribution of the contact resistance at the graphene-electrode interfaces [Fig. 11(b)]. This is important for the study of current-voltage characteristics as the contact resistance may change when increasing the applied voltage. In addition, the four-point configuration allows us to have a better spatial homogeneity of the carrier density. Indeed, the voltage electrodes can be patterned away from the central channel [Fig. 11(a)] so they screen the electric field between the gate and the graphene sheet to a lesser extent. The device is symmetrically voltage biased. The current is measured through electrodes $\mathrm{A}$ and $\mathrm{D}$ while the four-point voltage between electrodes $\mathrm{B}$ and $\mathrm{C}$.

Measurements are done at $300 \mathrm{~K}$ and $10^{-6}$ mbar. We take care not to degrade the graphene sheet when bringing the device into the high current regime. Indeed, these conditions are especially harsh, since a lot of energy (up to several milliwatts) is dissipated in a $\sim 1 \mu \mathrm{m}^{2}$ surface area. To prevent degradation, measurements are carried out in vacuum $\left(10^{-6} \mathrm{mbar}\right)$ and $I-V$ characteristics are measured rapidly (within $\sim 1 \mathrm{~s}$ ). After each measurement, we control that the gate-voltage dependence of the zero-voltage conductivity has not changed.

The mobility is obtained from the dependence of the conductivity on the gate voltage $V_{g}$ [see, e.g., Fig. 12(a)], as $\mu$ $=d \sigma /\left(d V_{g}\right) / C_{g}$, being $\sigma\left(V_{g}\right)$ the zero-bias conductivity as a function of $V_{g} . \mu$ is measured in the region where $\sigma\left(V_{g}\right)$ is linear (which is usually attributed to the presence of Coulomb scatterers ${ }^{19}$ ). The exponent $\alpha$ reported in the text are obtained from the linear fit of the $I-V$ in a double logarithmic scale plot for $V>0.1 \mathrm{~V}$.

\section{APPENDIX E: HIGH-MOBILITY DEVICES}

Measurements on high-mobility devices deserve a further discussion. Figure 12(b) shows the current-voltage character-
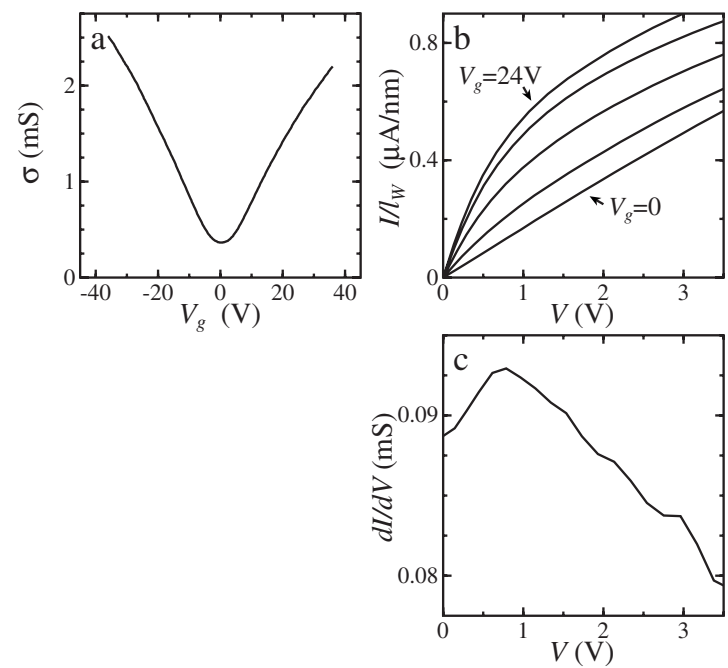

FIG. 12. Electronic transport in graphene: measurements on a high-mobility device $\left[7000 \mathrm{~cm}^{2} /(\mathrm{V} \mathrm{s})\right]$. (a) Conductivity as a function of the gate voltage $V_{g}$, measured at zero voltage $(V<k T)$. The width of the channel is $l_{W}=540 \mathrm{~nm}$ and the length is $l=2.1 \mu \mathrm{m}$. (b) $I$ - $V$ characteristics for different $V_{g}$ (from 0 to $24 \mathrm{~V}$ in $6 \mathrm{~V}$ steps). (c) Numerically differentiated $d I / d V$ as a function of $V$ at the Dirac point.

istics for several gate voltages measured on a device with a relatively high mobility of $7000 \mathrm{~cm}^{2} /(\mathrm{V} \mathrm{s})$. The $I-V$ appears linear at $V_{g}=0$ (Dirac point) which contrasts to the case of high $V_{g}$ for which the current tends to saturate. The current saturation has been carefully analyzed in Ref. 16 and has been attributed to scattering due to optical phonons of graphene $(0.15 \mathrm{~V})$. In some cases the relevant scattering can be due to phonons of the substrate with a smaller energy of $\simeq 50 \mathrm{meV} .{ }^{18} \mathrm{We}$ emphasize that the current saturation in Fig. 12(b) has a different origin from the one predicted in the case of ballistic graphene devices and shown in Fig. 2(d). There, the current saturation is a consequence that the nontunneling current ceases to increase when $e V>\epsilon_{F}$ (see Appendix A).

We now focus on the $I-V$ characteristics at the Dirac point. Careful inspection reveals a small deviation from linearity. This is better seen in Fig. 12(c) where the differential conductance $d I / d V$ is plotted as a function of $V . d I / d V$ does not remain constant but increases at low $V$ and decreases at $V$ above $\sim 1 \mathrm{~V}$. Similar results have been obtained for all the high-mobility devices that we have measured (eight in total).

The $I-V$ at the Dirac point of high-mobility devices can be interpreted as a consequence of the competition between the Zener tunneling effect and the contribution of the nontunneling intraband current, which tends to saturate. Namely, the superlinearity of the $I-V$ due to Zener tunneling is more or less compensated by the current saturation due to optical phonons. The balance between the two mechanisms changes when $V$ is increased. At low $V$, Zener-Klein tunneling dominates and the $I-V$ is slightly superlinear. At higher $V$, the scattering with optical phonons become more relevant and the $I-V$ is slightly sublinear, which is in agreement with the measurements in Fig. 12(c). Moreover, at high-bias self- 
heating of the graphene channel can be relevant. ${ }^{24}$ This is associated with an increase in the scattering with acoustic phonons resulting in a sublinear behavior which will also mask the ZK current.

As for low-mobility devices, the $I-V$ 's are observed to be much more superlinear. This is consistent with the picture of competition between Zener tunneling and scattering due to optical phonons. Indeed, disorder in low-mobility devices tends to suppress the saturation of the current ${ }^{16}$ (electrons are mainly scattered by disorder centers which reduce the effect of optical phonons on transport). As a result, the mechanism relevant for transport is Zener tunneling.
*Present address: Kavli Institute of Nanoscience Delft, Delft University of Technology, PO Box 5046, 2600 GA Delft, The Netherlands.

${ }^{1}$ C. Zener, Proc. R. Soc. London 145, 523 (1934).

${ }^{2}$ K. S. Novoselov, A. K. Geim, S. V. Morozov, D. Jiang, Y. Zhang, S. V. Dubonos, I. V. Grigorieva, and A. A. Firsov, Science 306, 666 (2004); Y. Zhang, Y.-W. Tan, H. L. Stormer, and P. Kim, Nature (London) 438, 201 (2005); K. S. Novoselov, A. K. Geim, S. V. Morozov, D. Jiang, M. I. Katsnelson, I. V. Grigorieva, S. V. Dubonos, and A. A. Firsov, ibid. 438, 197 (2005); A. H. Castro Neto, F. Guinea, N. M. R. Peres, K. S. Novoselov, and A. K. Geim, Rev. Mod. Phys. 81, 109 (2009).

${ }^{3}$ M. I. Katsnelson, K. S. Novoselov, and A. K. Geim, Nat. Phys. 2, 620 (2006).

${ }^{4}$ V. V. Cheianov and V. I. Fal'ko, Phys. Rev. B 74, 041403(R) (2006).

${ }^{5}$ M. M. Fogler, D. S. Novikov, L. I. Glazman, and B. I. Shklovskii, Phys. Rev. B 77, 075420 (2008); L. M. Zhang and M. M. Fogler, Phys. Rev. Lett. 100, 116804 (2008).

${ }^{6}$ E. B. Sonin, Phys. Rev. B 79, 195438 (2009).

${ }^{7}$ O. Klein, Z. Phys. 53, 157 (1929).

${ }^{8}$ M. C. Lemme, T. J. Echtermeyer, M. Baus, and H. A. Kurz, IEEE Electron Device Lett. 28, 282 (2007).

${ }^{9}$ B. Huard, J. A. Sulpizio, N. Stander, K. Todd, B. Yang, and D. Goldhaber-Gordon, Phys. Rev. Lett. 98, 236803 (2007).

${ }^{10}$ R. V. Gorbachev, A. S. Mayorov, A. K. Savchenko, D. W. Horsell, and F. Guinea, Nano Lett. 8, 1995 (2008).

${ }^{11}$ G. Liu, J. Velasco, W. Bao, and C. N. Lau, Appl. Phys. Lett. 92, 203103 (2008).

${ }^{12}$ N. Stander, B. Huard, and D. Goldhaber-Gordon, Phys. Rev. Lett. 102, 026807 (2009).

${ }^{13}$ A. F. Young and P. Kim, Nat. Phys. 5, 222 (2009).

${ }^{14}$ S. Datta, Quantum Transport: Atom to Transistor (Cambridge University Press, Cambridge, 2005).
${ }^{15}$ A. V. Andreev, Phys. Rev. Lett. 99, 247204 (2007).

${ }^{16}$ A. Barreiro, M. Lazzeri, J. Moser, F. Mauri, and A. Bachtold, Phys. Rev. Lett. 103, 076601 (2009).

${ }^{17}$ E. H. Hwang, S. Adam, and S. Das Sarma, Phys. Rev. Lett. 98, 186806 (2007); J. Martin, N. Akerman, G. Ulbricht, T. Lohnmann, J. H. Smet, K. von Klitzing, and A. Yakoby, Nat. Phys. 4, 144 (2008).

${ }^{18}$ J. Chen, C. Jang, S. Xiao, M. Ishigami, and M. S. Fuhrer, Nat. Nanotechnol. 3, 206 (2008); I. Meric, M. Y. Han, A. F. Young, B. Ozyilmaz, P. Kim, and K. L. Shepard, ibid. 3, 654 (2008).

${ }^{19}$ J. H. Chen, C. Jang, S. Adam, M. S. Fuhrer, E. D. Williams, and M. Ishigami, Nat. Phys. 4, 377 (2008); C. Jang, S. Adam, J. H. Chen, E. D. Williams, S. Das Sarma, and M. S. Fuhrer, Phys. Rev. Lett. 101, 146805 (2008); Y. W. Tan, Y. Zhang, K. Bolotin, Y. Zhao, S. Adam, E. H. Hwang, S. Das Sarma, H. L. Stormer, and P. Kim, ibid. 99, 246803 (2007).

${ }^{20}$ W. K. Tse, E. H. Hwang, and S. Das Sarma, Appl. Phys. Lett. 93, 023128 (2008).

${ }^{21}$ G. Stoltz, M. Lazzeri, and F. Mauri, J. Phys.: Condens. Matter 21, 245302 (2009).

${ }^{22}$ A. Bachtold, M. de Jonge, K. Grove-Rasmussen, P. L. McEuen, M. Buitelaar, and C. Schönenberger, Phys. Rev. Lett. 87, 166801 (2001).

${ }^{23}$ M. Bockrath, D. H. Cobden, J. Lu, A. G. Rinzler, R. E. Smalley, L. Balents, and P. L. McEuen, Nature (London) 397, 598 (1999).

${ }^{24}$ M. Freitag, M. Steiner, Y. Martin, V. Perebeinos, Z. Chen, J. C. Tsang, and P. Avouris, Nano Lett. 9, 1883 (2009).

${ }^{25}$ B. Dora and R. Moessner, Phys. Rev. B 81, 165431 (2010).

${ }^{26}$ B. Rosenstein, M. Lewkowicz, H. C. Kao, and Y. Korniyenko, Phys. Rev. B 81, 041416(R) (2010).

${ }^{27}$ J. Moser, A. Barreiro, and A. Bachtold, Appl. Phys. Lett. 91, 163513 (2007). 